\title{
Trial-to-trial size variability of motor-evoked potentials. A study using the triple stimulation technique
}

\author{
Kai M. Rösler · Denise M. Roth $\cdot$ Michel R. Magistris
}

Received: 23 November 2007 / Accepted: 8 January 2008 / Published online: 30 January 2008

(C) Springer-Verlag 2008

\begin{abstract}
Motor-evoked potentials (MEPs) vary in size from one stimulus to the next. The objective of this study was to determine the cause and source of trial-to-trial MEP size variability. In two experiments involving 10 and 14 subjects, the variability of MEPs to cortical stimulation (cortical-MEPs) in abductor digiti minimi (ADM) and abductor hallucis (AH) was compared to those responses obtained using the triple stimulation technique (corticalTST). The TST eliminates the effects of motor neuron (MN) response desynchronization and of repetitive $\mathrm{MN}$ discharges. Submaximal stimuli were used in both techniques. In six subjects, cortical-MEP variability was compared to that of brainstem-MEP and brainstem-TST. Variability was greater for MEPs than that for TST responses, by approximately one-third. The variability was the same for cortical- and brainstem-MEPs and was similar in ADM and AH. Variability concerned at least $10-15 \%$ of the MN pool innervating the target muscle. With the stimulation parameters used, repetitive $\mathrm{MN}$ discharges did not influence variability. For submaximal stimuli, approximately two-third of the observed MEP size variability is caused by the variable number of recruited alpha-MNs and approximately one-third by changing synchronization of MN discharges. The source of variability is most likely localized at the spinal segmental level.
\end{abstract}

K. M. Rösler ( $)$ · D. M. Roth

Department of Neurology, Inselspital,

Bern University Hospital and University of Bern,

3010 Bern, Switzerland

e-mail: kai-michael.roesler@insel.ch

M. R. Magistris

Department of Neurology, Hôpital Cantonal de Genève,

Geneva University, Geneva, Switzerland
Keywords Transcranial magnetic stimulation . Brainstem magnetic stimulation · Clinical neurophysiology . Response variability

\section{Introduction}

Transcranial magnetic stimulation (TMS) induces muscle responses termed motor-evoked potentials (MEPs). A characteristic of MEPs is that their size and shape varies from one stimulus to the next, even if the stimulus parameters are kept constant (Kiers et al. 1993; Woodforth et al. 1996; Ellaway et al. 1998). A number of mechanisms may account for this variability, including:

1. changes of excitability of the target cortical or anterior horn motor neurons (MNs), resulting in the activation of a variable number of spinal MNs;

2. variation of the synchronization of the $\mathrm{MN}$ discharges, causing varying phase cancellation;

3. varying occurrence of repetitive alpha-MN discharges (repMNDs) in response to the brain stimuli.

The triple stimulation technique (TST) eliminates the influence of the spinal MN discharge desynchronization and of the repMNDs by means of a collision technique (Magistris et al. 1998). Using this technique we showed that in healthy subjects, nearly $100 \%$ of spinal MNs innervating a small hand (Magistris et al. 1998) or an intrinsic foot muscle (Bühler et al. 2001) could be brought to discharge by TMS. At the same time, the variability of size of the resynchronized responses was remarkably small, thus emphasizing the possible influence of changes of MN discharge synchronization and of repMNDs on the MEP size variability.

In our previous experiments, we studied maximal responses, where virtually $100 \%$ of MNs were activated. In 
such a setting, the variability of the responses may be reduced by a "saturation" or "ceiling" effect, i.e., if the stimulation strength is well above the threshold of all involved MNs, the variability will be low. In the present study, we investigated the variability of responses elicited by submaximal TMS. We compared the variability of conventional MEPs with that of TST responses (obtained with identical stimulation parameters) to sort out the effects of varying MN discharge desynchronization and repMNDs (avoided by the TST) versus that of changing numbers of excited MNs. In addition, to locate the source of variability, we performed stimulation at the level of the brainstem, by using the method described by Ugawa et al. (1994) (brainstem-MEPs), and by combining it with a TST protocol (brainstem-TST).

\section{Materials and methods}

\section{Subjects}

Two sets of experiments were performed. In Experiment I, 10 healthy subjects were examined, 3 women and 7 men, aged 20-43 years. In Experiment II, 14 different healthy subjects were studied, 10 women and 4 men, aged 22-26 years. None of them had a history of neurological disorder, implanted metallic material in the eye or brain, or a cardiac pacemaker. The study was approved by the local ethics committee, and informed consent was obtained from all subjects.

Electrophysiological and mechanical recordings

Compound muscle action potentials (CMAPs) were recorded from the abductor digiti minimi (ADM) or the abductor hallucis muscle (AH) using the muscle-belly tendon technique with surface electrodes (diameter $0.8 \mathrm{~cm}$ ). A ground electrode was taped to the dorsum of the hand or over the heel. A Viking Select EMG apparatus was used for the recordings (Viasys Biomedical, Madison, WI, US.). Bandpass filtering was $2 \mathrm{~Hz}-10 \mathrm{kHz}$. We used the software package "emgepview32" (A. Broutian, http://www.broutian.dol.ru) to digitally extract and analyze the curve signals from the Viking apparatus (the curves depicted in Fig. 2 were obtained using this program). For ADM recordings, the subjects sat on a chair with their left forearm and hand fastened with Velcro straps on a platform in front of them. To determine the force of voluntary muscle contraction, the fifth finger was attached to a force transducer (Sensotec, Inc., OH, USA). The force signal was amplified using a Sedia DC amplifier (Sedia, Givisiez, Switzerland), and digitized by a MacLab stand-alone AD-converter (ADInstruments Pty Ltd., Castle Hill, NSW, Australia). It was then presented on a digital oscilloscope in front of the subject to allow for a visual feedback (Arányi et al. 1998). The maximal voluntary contraction force (MVC) was measured, and the target force levels (either 5 or $20 \%$ of MVC, see subsequent section) were indicated as goals marked on the oscilloscope screen.

In Experiment II, recordings from $\mathrm{ADM}$ and $\mathrm{AH}$ were obtained. These recordings were always done during muscle relaxation, so that force recordings were not necessary. For AH measurements, subjects lay prone with the feet comfortably placed on a round cushion (Bühler et al. 2001). ADM recordings were conducted as in Experiment I, with the exception of force measurements.

\section{Magnetic stimulation}

Transcranial magnetic stimulation was performed using a Magstim 200 stimulator (Magstim Company Ltd., SpringGardens, Whitland, UK). For cortical stimulation of the left $A D M$, a $90 \mathrm{~mm}$ circular coil was centered over the vertex or slightly lateral toward the stimulated right hemisphere. Face 'B' was up. For cortical stimulation of the AH, a double cone figure-of- 8 coil was used, with the center of the " 8 " overlying the vertex. Slight coil displacements were made in all directions until the position yielding the lowest threshold was found. The optimal position was marked on the scalp, and the coil was then kept in the same position throughout the experiment. The resting threshold was determined as the stimulation intensity in percent of maximum stimulator output that yielded a discernible response of the relaxed target muscle in $50 \%$ of 8 or 10 trials at an amplification of $100 \mu \mathrm{V} /$ division (Rothwell et al. 1999). For brainstem stimulation of $A D M$, a double cone coil was used in a position as described by Ugawa et al. (1994). In most subjects, the threshold to elicit a brainstem-evoked response was near $100 \%$ of the stimulator output. Brainstem stimulation of $\mathrm{AH}$ was not performed.

Triple stimulation technique

The TST has previously been described in detail (Magistris et al. 1998, 1999; Bühler et al. 2001). In short, it consists of a succession of three stimuli. For recordings from ADM, the following stimuli are given: (1) the transcranial magnetic brain stimulus, (2) a maximal stimulus to the ulnar nerve at the wrist, and (3) a maximal stimulus to the brachial plexus at Erb's point (Magistris et al. 1998). To account for the possible volume conduction by the median nerve (which may be co-stimulated by the Erb stimulus), we stimulated both, ulnar and median nerve at the wrist (for a detailed discussion of volume conduction in the TST, see Humm et al. 2004). For recordings from AH, the stimuli are: (1) the transcranial magnetic brain stimulus, (2) a maximal stimulus to the tibial nerve at the ankle, and (3) a 
maximal stimulus to the sciatic nerve at the level of the gluteal fold, applied through a needle placed close to the nerve (Bühler et al. 2001; Yap and Hirota 1967). For brainstemTST, the cortical stimulus was replaced by a brainstem stimulus (Ugawa et al. 1994). The stimuli were appropri-

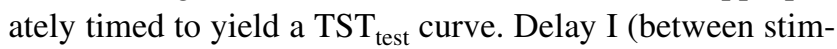
uli 1 and 2) was calculated by subtracting the latencies of the wrist stimulus from that of the brain stimulus and rounded down to the nearest millisecond. Delay II (between stimuli 2 and 3) was calculated by subtracting the latency of the Erb stimulus minus that of the wrist stimulus and rounded down to the nearest millisecond. The events occurring as a result of the TST are depicted in Fig. 1. The cortical-TST $_{\text {test }}$ curve or brainstem-TST test $_{\text {curve was compared }}$ to a $\mathrm{TST}_{\text {control }}$ curve, obtained by three successive stimuli given at (1) the Erb's point, (2) the ulnar nerve at the wrist, and (3) the Erb's point, or at (1) the sciatic nerve at the gluteal fold, (2) the tibial nerve at the ankle, and (3) the sciatic nerve at the gluteal fold (Fig. 1). The amplitude ratio of $\mathrm{TST}_{\text {test }}: \mathrm{TST}_{\text {control }}$ (termed TST amplitude ratio) reflects the



A1 a) $\mathrm{TST}_{\text {test }}$

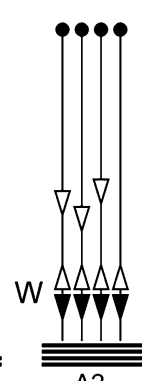

$\mathrm{A} 2$

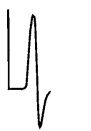

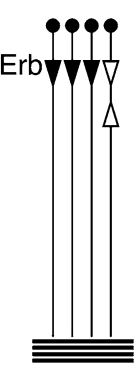

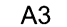

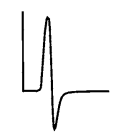

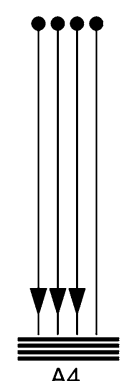

$\| \sqrt{ }$ proportion of spinal MNs innervating the target muscle brought to discharge by the TMS (Magistris et al. 1998).

\section{Experimental protocol}

\section{Experiment I}

The individual resting threshold and the optimal coil position for cortical-TMS were determined. The resting threshold stimulation strength was used for all subsequent brain stimuli. The individual TST delays were determined by peripheral stimulation of the ulnar nerve at the wrist and at Erb's point. MVC force of little finger abduction was determined in three trials. Amplitude and area of the CMAP evoked by maximal Erb stimulation $\left(\mathrm{CMAP}_{\mathrm{Erb}}\right)$ were determined during voluntary contractions of 5 and $20 \%$ of MVC. Then, 24 MEPs and $24 \mathrm{TST}_{\text {test }}$ responses were sampled during voluntary contractions of 5 and $20 \%$ of MVC. The $\mathrm{TST}_{\text {test }}$ sampling was preceded and followed by recording of a TST $_{\text {control }}$ curve at 5 or $20 \%$ of MVC. Finally, eight



B1 b) TST $_{\text {control }}$

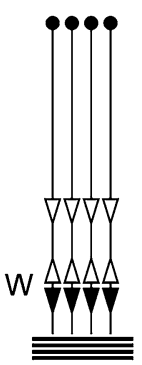

B2

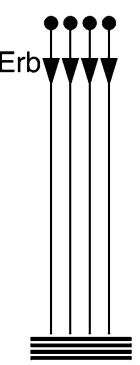

B3



B4


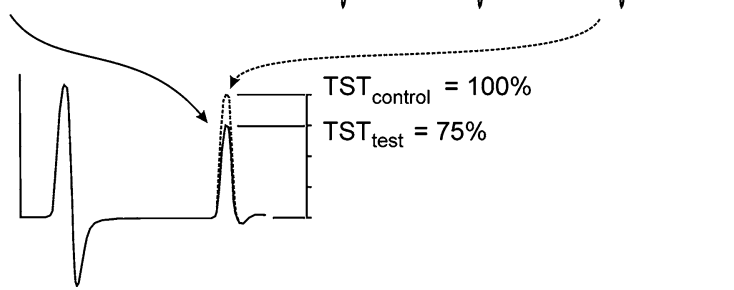

Fig. 1 Triple stimulation technique (TST) principle, recording from abductor digiti minimi (ADM). The motor tract is simplified to four spinal motor neurons (MNs); horizontal lines represent the muscle fibers of the four motor units of ADM. Open arrows indicate action potentials (APs) that are not recorded in the resulting trace and solid arrows contribute to the recorded trace. In this example, a submaximal transcranial stimulus excites three out of four spinal MNs (large open arrows). Desynchronization of the three APs has occurred. A1 Transcranial stimulation excites three out of four MNs; (A2) after a delay, a maximal stimulus is given to the ulnar nerve at the wrist (W) and evokes a first negative deflection of the recording trace. The antidromic APs collide with the descending APs on MNs 1-3, but continues to ascend on MN 4. A3 After a second delay, a maximal stimulus is given at Erb's point. On MN 4, the descending AP collides with the ascending AP. A4 A synchronized response from the three MNs that were initially excited by the transcranial stimulus is recorded as the second deflection of the $\mathrm{TST}_{\text {test }}$ trace. The $\mathrm{TST}_{\text {control }}$ curve is obtained with the following stimuli: (B1) A maximal stimulus is given at Erb's point; (B2) after a delay, a maximal stimulus applied to the ulnar nerve at the wrist $(W)$ evokes the first deflection of the $\mathrm{TST}_{\text {control }}$ trace; $(B 3)$ after a delay a maximal stimulus is given at Erb's point; $(B 4)$ a synchronized response from the four MNs is recorded as the second deflection of the $\mathrm{TST}_{\text {control }}$ trace. The TST amplitude ratio is measured as depicted in the figure. It corresponds to the proportion of MNs that were driven to discharge by the TMS 
brainstem-MEP responses and eight brainstem-TST responses were sampled; both using identical stimulation intensities of $80-100 \%$ of the maximal stimulator output and a background contraction of $20 \%$ of MVC.

\section{Experiment II}

Recordings from ADM and AH were obtained in each subject. The target muscles were relaxed during stimulations. First, ADM was examined, followed by examination of $\mathrm{AH}$. We attempted to use a stimulation intensity that brought $50 \%$ of the target $\mathrm{MN}$ pool to discharge (i.e., yielding a TST amplitude ratio of 50\%). Once the appropriate stimulation strength was defined, 12 TST recordings and 12 MEP recordings were performed in a random sequence.

\section{Statistical methods}

The variability of responses was calculated from 12 consecutive stimuli for all brain stimulation studies and from 8 consecutive stimuli for the brainstem stimulation studies. Calculation from 12 responses was judged to be sufficient, because results calculated for 24 stimuli in Experiment I were virtually identical to those calculated from 12 stimuli, and results of the first 12 stimuli were identical to those of the second 12 stimuli. Two parameters were used as measures of variability, namely the coefficient of variation (CV) and the mean consecutive difference (MCD), as suggested by Kiers et al. (1993). The CV is the standard deviation divided by the mean. The MCD is the mean of differences between subsequent stimuli. To allow a comparison between MCD of MEP and TST responses, the MCD was normalized to the average size of the responses.

The $\mathrm{TST}_{\text {test }}: \mathrm{TST}_{\text {control }}$ ratio measures the proportion of spinal MNs brought to discharge by TMS (Magistris et al. 1998). By analogy, we calculated the proportion of spinal MNs subject to variability (i.e., MNs that did or did not discharge) during successive stimuli of a given stimulus modality. This was done by normalizing the MCD of TST responses to $\mathrm{TST}_{\text {control }}\left(\mathrm{TST}_{\mathrm{MCD}}: \mathrm{TST}_{\text {control }}\right)$.

Nonparametric tests were used to test the differences between group means. The level of significance was set at $P<0.05$.

\section{Results}

\section{Response size}

In both experiments, there was a great variability in the average MEP and TST response size between subjects, despite the standardized stimulation parameters (stimulation at the individually determined resting threshold inten- sity, monitored background contraction; or stimulation of $50 \%$ of the MN pool). Despite these inter-individual differences in response size, the TST responses were larger than the MEP responses obtained under equal stimulation conditions (Table 1). The mean MEP:TST ratio was 71 and $75 \%$ in Experiment I, where responses were facilitated by a background contraction of the target ADM. This ratio is in good agreement with the previously published data (Magistris et al. 1998, 1999; Rösler et al. 2000). In Experiment II, we attempted to obtain similar-sized responses from all subjects and stimulated with a stimulation intensity yielding a TST amplitude ratio of roughly 50\% in each subject with relaxed target muscle. This response required much higher stimulation intensities (Table 1). It was not always possible to reach 50\% TST amplitude ratio in the relaxed muscle, because $100 \%$ stimulator output was not always sufficient. On average, however, the TST amplitude ratio reached the goal of $50 \%$ (Table 1).

In Experiment II, the MEP:TST ratio was 38\% in both muscles (Table 1). The smaller ratio is probably explained by the fact that stimulations were done with the relaxed target muscles.

All subjects judged brainstem stimulation as rather unpleasant. The threshold for brainstem stimulation was always much higher than that for cortical stimulation, such that responses at $20 \%$ MVC were never obtained with stimulator intensities below $80 \%$. In three subjects, brainstem stimulation did not yield any responses despite use of maximal stimuli, and one subject did not give his consent to this procedure. The average response size in the remaining six subjects was in a similar range than that with cortical stimulation at 5\% MVC (Table 1). Differences between brainstem-TST response size and brainstem-MEP size were not as great as with cortical stimulation (Table 1), and in two out of six subjects, brainstem-MEPs were even larger than brainstem-TST responses, probably explained by an effect of response variability during the sampling of responses.

\section{Relative MEP versus TST variability}

Both MEP and TST responses varied in size from one stimulus to the next (Fig. 2). To compare the variability between MEPs and TST, two measures of the relative variability of responses, the $\mathrm{CV}$ and the normalized MCD, were used. In all experiments, these parameters correlated well with one another (linear regression $r^{2}$ between 0.71 and 0.94; all $P<0.0003$ ). Variability differed considerably between individuals. The average relative variability of MEP responses was larger than that of TST responses in all stimulation paradigms (Tables 2, 3). The difference in variability of MEP versus TST was around one-third, which is smaller than that anticipated from previous studies and did not reach statistical significance in the leg muscle (Table 3) 
Table 1 Stimulation intensity and response size in the two experiments comparing MEP and TST variability
Given are inter-individual averages \pm standard deviation and [range]

$A D M \mathrm{~m}$. abductor digiti minimi, $A H \mathrm{~m}$. abductor hallucis

\begin{tabular}{|c|c|c|c|c|}
\hline & Response size & Range & Stimulus intensity & Range \\
\hline \multicolumn{5}{|l|}{ Experiment I } \\
\hline \multicolumn{5}{|c|}{ ADM 5\% background contraction ( $n=10$ subjects) } \\
\hline $\operatorname{MEP}(\mathrm{mV})$ & $2.7 \pm 1.3$ & {$[0.9-4.8]$} & \multirow[t]{4}{*}{$44 \pm 6 \%$} & \multirow[t]{4}{*}[35-55\%]{} \\
\hline $\mathrm{TST}(\mathrm{mV})$ & $3.8 \pm 1.4$ & {$[1.3-6.1]$} & & \\
\hline TST amplitude ratio & $47 \pm 17 \%$ & {$[19-69 \%]$} & & \\
\hline MEP/TST ratio & $71 \pm 22 \%$ & [29-112\%] & & \\
\hline \multicolumn{5}{|c|}{ ADM $20 \%$ background contraction ( $n=10$ subjects) } \\
\hline MEP (mV) & $4.2 \pm 1.0$ & {$[2.9-5.5]$} & \multirow[t]{4}{*}{$44 \pm 6 \%$} & \multirow[t]{4}{*}[35-55\%]{} \\
\hline TST (mV) & $5.7 \pm 1.6$ & {$[3.8-8.4]$} & & \\
\hline TST amplitude ratio & $73 \pm 18 \%$ & {$[43-91 \%]$} & & \\
\hline MEP / TST ratio & $75 \pm 6 \%$ & {$[65-87 \%]$} & & \\
\hline \multicolumn{5}{|c|}{ ADM $20 \%$ background contraction—brainstem stimulation ( $n=6$ subjects) } \\
\hline $\mathrm{MEP}(\mathrm{mV})$ & $2.5 \pm 0.8$ & {$[1.5-3.5]$} & \multirow[t]{4}{*}{$97 \pm 8 \%$} & \multirow{4}{*}[80-100\%]{} \\
\hline TST (mV) & $2.9 \pm 0.7$ & {$[1.7-3.8]$} & & \\
\hline TST amplitude ratio & $39 \pm 7 \%$ & {$[29-45 \%]$} & & \\
\hline MEP / TST ratio & $89 \pm 18 \%$ & [71-119\%] & & \\
\hline \multicolumn{5}{|l|}{ Experiment II } \\
\hline \multicolumn{5}{|c|}{ ADM $0 \%$ background contraction ( $n=14$ subjects $)$} \\
\hline $\mathrm{MEP}(\mathrm{mV})$ & $1.8 \pm 0.9$ & {$[0.8-3.8]$} & \multirow[t]{4}{*}{$75 \pm 22 \%$} & \multirow[t]{4}{*}[50-100\%]{} \\
\hline $\mathrm{TST}(\mathrm{mV})$ & $4.8 \pm 1.7$ & {$[2.4-9.4]$} & & \\
\hline TST amplitude ratio & $51 \pm 10 \%$ & {$[36-71 \%]$} & & \\
\hline MEP/TST ratio & $38 \pm 18 \%$ & {$[17-72 \%]$} & & \\
\hline \multicolumn{5}{|c|}{ AH $0 \%$ background contraction ( $n=14$ subjects) } \\
\hline $\operatorname{MEP}(\mathrm{mV})$ & $1.7 \pm 1.0$ & {$[0.4-4.3]$} & \multirow[t]{4}{*}{$81 \pm 16 \%$} & \multirow[t]{4}{*}[50-100\%]{} \\
\hline $\mathrm{TST}(\mathrm{mV})$ & $4.8 \pm 2.0$ & [1.9-7.9] & & \\
\hline TST amplitude ratio & $49 \pm 19 \%$ & {$[22-77 \%]$} & & \\
\hline MEP/TST ratio & $38 \pm 19 \%$ & {$[12-78 \%]$} & & \\
\hline
\end{tabular}

and in the 5\%-background contraction paradigm in Experiment I (Table 2).

In Experiment I, variability was somewhat larger for the responses elicited during background contractions of $5 \%$ MVC than that of $20 \%$ background contraction (Table 2). Response variability in Experiment II (no background contraction) was, on average, smaller than that in Experiment I. Variability in AH tended to be smaller than that in ADM, but this difference did not reach statistical significance due to the great inter-individual differences.

Relative variability of brainstem versus cortical stimulation responses

The CV and normalized MCD of brainstem stimulation responses were not statistically different than those of cortical stimulation (Table 2).

\section{Absolute response variability}

Using the TST, the percentage of MNs of the MN pool varying from stimulus to stimulus could be quantified by the ratio of $\mathrm{TST}_{\mathrm{MCD}}$ : $\mathrm{TST}_{\text {control }}$ which was between 10 and $15 \%$ with our stimulation paradigms (Tables 2, 3). Thus, $10-15 \%$ of the MN pool supplying the target muscle was subject to stimulus-to-stimulus variability. This ratio probably underestimates the proportion of the MN pool that may or may not discharge, because the trial-to-trial difference between two stimuli does not include all MNs that may or may not discharge after a given stimulus (i.e., the pool of MNs not responding is greater than 10-15\%). Our data thus suggest that at least $10-15 \%$ of the entire MN pool of the target muscles was very close to threshold ("fringe MNs"), and that this proportion was largely independent of the stimulation intensity, of the size of the response, and of the site of stimulation (i.e., whether following cortical or brainstem stimulation). Our data also did not demonstrate a difference between $\mathrm{ADM}$ and $\mathrm{AH}$ in this respect.

\section{Discussion}

Muscle responses to TMS vary in size from one stimulus to the next. In this study, we analyzed the differential effects of 
Fig. 2 Complete recordings in one subject, Experiment I. To demonstrate the amplitude variability, only the main deflections of the traces are shown. This reduction eliminates the first deflection of the TST curves. Variability was calculated from the first subsequent 12 responses; 24 responses are shown. The muscle response to Erb stimulation $\left(\mathrm{CMAP}_{\mathrm{Erb}}\right)$ and the TST $\mathrm{T}_{\text {con- }}$ trol curve are given on the left to allow appreciation of the relative size of the MEP and TST responses
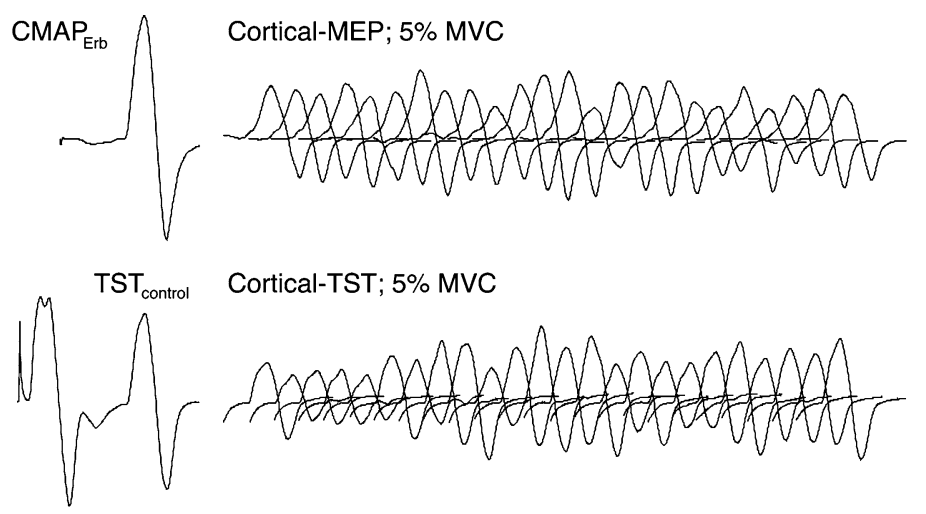

Brainstem-MEP; 20\% MVC

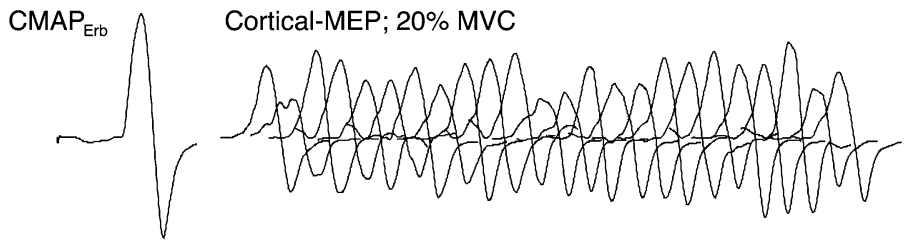

TST $_{\text {control }}$ Cortical-TST; 20\% MVC

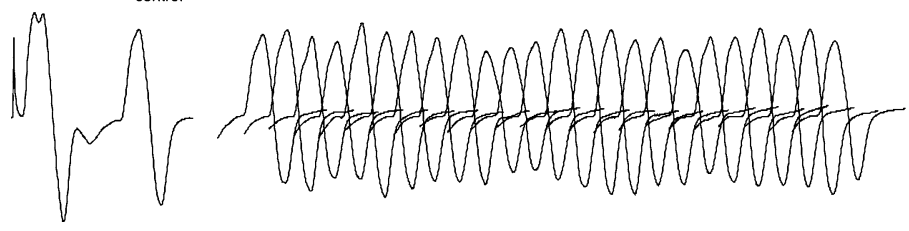

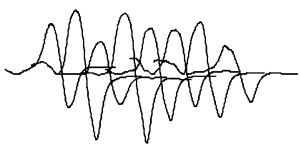

Brainstem-TST; 20\% MVC

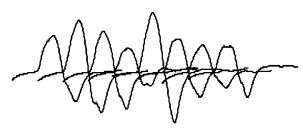

Table 2 Experiment I: response variability of MEP and TST amplitudes in M. abductor digiti minimi

\begin{tabular}{|c|c|c|c|c|c|}
\hline $\begin{array}{l}\text { Stimulation } \\
\text { site }\end{array}$ & $\begin{array}{l}\text { Background } \\
\text { contraction }\end{array}$ & Parameter & MEP & TST & Wilcoxon $P$ \\
\hline Cortex & $5 \% \mathrm{MVC}$ & $\begin{array}{l}\text { Response amplitude [mV] } \\
\text { Coefficient of variation [\%] } \\
\mathrm{MCD} \text { in \% of response amplitude [\%] } \\
\mathrm{MCD} \text { in \% of } \mathrm{TST}_{\text {control }}\end{array}$ & $\begin{array}{l}2.67 \pm 1.29 \\
40.5 \pm 21.5 \% \\
42.2 \pm 17.0 \%\end{array}$ & $\begin{array}{l}3.75 \pm 1.44 \\
32.2 \pm 21.5 \% \\
37.0 \pm 20.8 \% \\
15.3 \pm 6.7 \% *\end{array}$ & $\begin{array}{l}P=0.007 \\
\mathrm{NS} \\
\mathrm{NS}\end{array}$ \\
\hline Cortex & $20 \% \mathrm{MVC}$ & $\begin{array}{l}\text { Response amplitude }[\mathrm{mV}] \\
\text { Coefficient of variation [\%] } \\
\mathrm{MCD} \text { in \% of response amplitude [\%] } \\
\mathrm{MCD} \text { in } \% \text { of } \mathrm{TST}_{\text {control }}\end{array}$ & $\begin{array}{l}4.23 \pm 1.03 \\
22.7 \pm 9.7 \% \\
22.8 \pm 9.6 \%\end{array}$ & $\begin{array}{l}5.71 \pm 1.62 \\
13.4 \pm 6.2 \% \\
14.7 \pm 7.0 \% \\
10.0 \pm 4.0 \% *\end{array}$ & $\begin{array}{l}P=0.005 \\
P=0.02 \\
P=0.04\end{array}$ \\
\hline Brainstem & $20 \% \mathrm{MVC}$ & 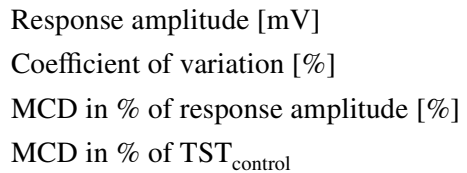 & $\begin{array}{l}2.55 \pm 0.78 \\
38.3 \pm 9.4 \% \\
48.9 \pm 11.1 \%\end{array}$ & $\begin{array}{l}2.85 \pm 0.69 \\
37.1 \pm 5.3 \% \\
43.2 \pm 11.1 \% \\
16.2 \pm 3.1 \%\end{array}$ & $\begin{array}{l}P=0.03 \\
P=0.03 \\
P=0.03\end{array}$ \\
\hline
\end{tabular}

Variability parameters are given in relation to two different degrees of precontraction (5 and 20\% of maximal voluntary contraction), and for cortical versus brainstem stimulation. Cortical stimulation with resting threshold stimulation intensity, brainstem stimulation intensity between 80 and $100 \%$ of maximal stimulator output. Coefficient of variation and mean consecutive difference (MCD) are calculated from 12 consecutive responses (brainstem: 8 consecutive responses). Given are means and SD of 10 subjects (brainstem: 6 subjects)

* No statistically significant difference between 5 and $20 \%$ precontraction (Wilcoxon)

three independent factors on this trial-to-trial size variability, namely the variation of the number of motor units responding to a given stimulus, the variation of the synchronization of the TMS induced MN discharges, and the variation of repMNDs. MN discharge desynchronization and repMNDs have previously been shown to markedly influence the size of MEPs (Magistris et al. 1998). For our analysis, we compared conventional MEPs (i.e., responses composed of desynchronized motor unit discharges and repMNDs) with TST responses (i.e., "resynchronized" TMS responses without repMNDs), obtained using identical stimulation modalities. We found that MEP size vari- 
Table 3 Experiment II: response variability of MEP and TST amplitudes in two muscles

\begin{tabular}{lllll}
\hline Muscle & Parameter & MEP & TST & Wilcoxon $P$ \\
\hline ADM & Response amplitude [mV] & $1.77 \pm 0.90$ & $4.84 \pm 1.66$ & $P=0.001$ \\
& Coefficient of Variation [\%] & $35.1 \pm 18.0 \%$ & $24.2 \pm 10.6 \%$ & $P=0.02$ \\
& MCD in \% of response amplitude [\%] & $39.0 \pm 21.8 \%$ & $26.3 \pm 15.0 \%$ & $13.5 \pm 10.6 \% *$ \\
& MCD in \% of TST control $^{*}$ & & $4.81 \pm 1.97$ & $P .016$ \\
AH & Response amplitude [mV] & $1.69 \pm 0.96$ & $18.5 \pm 9.6 \%$ & NS \\
& Coefficient of Variation [\%] & $22.1 \pm 10.1 \%$ & $20.1 \pm 10.7 \%$ & $8.4 \pm 3.7 \% *$ \\
\hline
\end{tabular}

Relaxed target muscles stimulation intensity such as to yield a TST amplitude ratio of 50\%. Coefficient of variation and mean consecutive difference (MCD) are calculated from 12 consecutive responses. Given are means and SD of 14 subjects

* No statistically significant difference between ADM and AH (Wilcoxon)

ability was greater than TST variability by approximately one-third (Tables 2, 3). It is unlikely that repMNDs influenced variability in the present set of experiments (see below). Thus, one-third of the variability of the MEP size was caused by variation of synchronization and two-thirds by variation of the number of activated motor units.

The present study extends the findings of Magistris and coworkers (1998) on the variability of TST responses. In a small variability study of MEP and TST responses in ADM of five subjects, these authors reported a CV of $8.1 \%$ for MEP amplitudes and of $2.6 \%$ for TST amplitudes. From their data, a $\mathrm{TST}_{\mathrm{CV}}$ : $\mathrm{TST}_{\text {control }}$ of $2.7 \%$ can be calculated; hence the $\mathrm{TST}_{\mathrm{MCD}}$ : $\mathrm{TST}_{\text {control }}$ was probably some 3\% (MCD was not given in that study and the original data were not available; however, MCD is somewhat larger than the CV, see Tables 1, 2, 3). Therefore, the absolute response variability measured by Magistris and coworkers was considerably lower than the $10-15 \%$ found here (see Tables 1, 2, 3). As mentioned in the Introduction, this is probably caused by a "ceiling" or "saturation" effect. Magistris et al. attempted and succeeded to stimulate the entire MN pool of the target muscle by using high stimulation intensities and ample facilitation maneuvers (i.e., "maximal" or "supramaximal" stimulation). The average stimulation intensity in their five subjects was $76 \%$ and facilitation by target muscle background contraction was used in all subjects, whereas in the present study, the stimulation intensity was $44 \%$ in the trials using facilitation, or $75 \%$ in the trials without facilitation (Table 1). The average TST amplitude ratio obtained in their five subjects was $97.2 \%$, thus representing the response of virtually all MNs in that study. In the present study, the average TST amplitude ratios were between 39 and $73 \%$ (Table 1), thus "submaximal". If the TMS stimulation intensity is well above the threshold of all involved MNs (or if facilitation maneuvers lower the threshold of all MNs), a saturation effect occurs, and variability is low. On the other hand, MEPs are probably less affected by this effect, because variability of discharge synchronization still occurs with maximal stimuli, causing MEPs to vary even when all MNs are brought to discharge by the stimulus. This explains the much larger difference between MEP and TST variability in the study of Magistris et al. compared with the present study. It is noteworthy that the relative measures of MEP variability (i.e., the CV) cannot directly be compared in the two studies, because it depends on the response size, which differs between the studies.

In the present study, we attempted to minimize methodological factors influencing response variability. A possible source of MEP variability is the influence of varying degrees of target muscle relaxation or background contraction (Hess et al. 1986; Kischka et al. 1993). Therefore, we standardized the degree of voluntary background contraction to 0,5 , and $20 \%$ of the individual maximal voluntary force, by measuring the force of little finger abduction during TMS. It is thus unlikely that varying degrees of facilitation by voluntary muscle contraction influenced our results. Moreover, particular care was taken to keep the position of the stimulating coil constant, by marking the coil position on the head of the subjects and readjusting the coil position following every stimulus. This was judged superior to a coil fixation device, because the Erb stimulations involved in the TST may lead to head movements by activation of neck muscles. Nevertheless, small variations in coil positioning might have occurred, theoretically contributing to the response variability. This factor is probably small, and previous studies have shown that the contribution of varying the coil position cannot account for the observed MEP variability (Ellaway et al. 1998; Gugino et al. 2001). For responses to cortical stimulation, the response variability measured in the present study is thus likely to be a result of an intrinsic mechanism inherent to TMS of the corticospinal pathway. In contrast to cortical stimulation, we found it more difficult to maintain a constant coil position for brain- 
stem stimulation. Here, small changes of coil position appeared to be more critical in influencing the response size. Moreover, brainstem stimuli usually induced rather strong contractions of head and neck muscles, disturbing the positioning of the stimulation coil. Hence, variability of brainstem-MEP and brainstem-TST may have been influenced by coil movements, even though we did our best to keep the stimulation geometry constant between the stimuli.

One conceivable source of response variability of MEPs (but not of TST responses) is the possibility of varying amounts of repMNDs in response to consecutive transcranial stimuli. RepMNDs occur often after single-shock TMS (Berardelli et al. 1991; Magistris et al. 1998; Naka et al. 2000; Z'Graggen et al. 2005). They may influence the size of an MEP, but not that of the TST response, because repetitive discharges escape the collisions involved in the TST procedure (Magistris et al. 1998; Bühler et al. 2001; Z'Graggen et al. 2005). Thus, if varying amounts of repMNDs occurred in the present experiments, they might have increased MEP variability, but not TST variability. RepMNDs are an indirect consequence of the nature of the descending corticospinal volley after TMS, which contains a succession of excitatory waves (termed D- and I-waves). These converge on the spinal MN (Patton et al. 1954; Day et al. 1987; Berardelli et al. 1991), eventually causing it to discharge repetitively (Day et al. 1989). Using cervical epidural recordings in patients undergoing spinal surgery, the variability of I-waves was found to be considerable (Burke et al. 1995) and was held responsible for at least some of the observed MEP variability (Ellaway et al. 1998). In the present experiments, an influence of varying amounts of repMNDs on MEP size variability can be excluded. First, brainstem stimulation does not evoke multiple descending corticospinal volleys (Ugawa et al. 1994); yet the response variability to brainstem stimuli was not smaller than with cortical stimuli (Fig. 2; Table 2). Second, repMNDs have a high threshold, and notable amounts of repMNDs occur only when some $75-80 \%$ of all MNs are discharging after TMS (yielding a TST amplitude ratio of 75-80\%; Z'Graggen et al. 2005). In the present study, stimulation intensities were thus not sufficient to evoke repMNDs. Taken together, the present data are not compatible with an important influence of repMNDs on MEP response variability. Repetitive discharges might however have influenced the MEP variability results of Magistris et al. (1998), who stimulated with maximal stimulation intensities yielding TST amplitude ratios near $100 \%$.

Our data give additional insight into the mechanisms involved in producing the MEP variability. First, the variability was roughly the same for brainstem and cortical stimulation. Variability was also similar in a small hand muscle and a foot muscle (see Tables 1, 2, 3). While small hand muscles are under the strong influence of direct monosynaptic corticospinal input, foot muscles are controlled to a greater extent by spinal reflex mechanisms, yet variability was similar. Both observations suggest that the main source of variability was not at the cortical level. Instead, varying recruitment of spinal MNs by the TMS induced descending activity is a more likely mechanism. This is supported by the observation of Woodforth and coworkers (1996). Using epidural recordings, they showed that the variability of descending volleys following brain stimulation is smaller than that of the MEP in a leg muscle, suggesting a mechanism distal to the cervical recording site. Our conclusion differs from that of other authors. Kiers et al. (1993) were able to compare the variability of the $\mathrm{H}$-reflex with that of a comparatively sized MEP in the thenar muscle of one subject and found less trial-to-trial variability of the H-reflex. Since the H-reflex is generally thought to reflect spinal MN excitability, they concluded that the source of MEP variability had to be supraspinal. It is a general observation that H-reflex variability is less than the variability observed here for MEPs and TST responses (Schieppati et al. 1990; Jankus et al. 1994). However, it is not certain that the MN pool affected by the muscle spindle primary afferents involved in the H-reflex is the same as that targeted by TMS. Summarized, while our data do not rule out some degree of corticospinal variability to play a role in certain situations, they are well compatible with an important spinal segmental source of MEP size variability.

While other studies quantified the relative variability of responses to TMS, this study is the first that gives an estimate of the proportion of the MN pool that may or may not respond to TMS. This was possible by using the TST, which-in contrast to MEPs-allows a quantification of the MNs responding to the brain stimulus (Magistris et al. 1998). Our data suggest that the trial-to-trial variability concerned well over 10-15\% of the entire MN pool of the target muscles (see Tables 1, 2, 3). These "fringe" MNs are very close to the firing threshold, such that small fluctuations in their membrane threshold may or may not suffice to lead them to respond to the corticospinal volley induced by the TMS. Remarkably, this proportion appeared rather uniform in all stimulation paradigms including those with or without facilitation by muscular background contraction and stimulations using different intensities. It is tempting to speculate about the cause of the fluctuation of the threshold of spinal MNs leading them to respond unpredictably to TMS. The present study gives no hint as to this cause. It is possible that small differences in arousal or mental activity may be sufficient to influence MNs close to threshold, as hypothesized previously by others (Rossini et al. 1991; Ellaway et al. 1998), and by us (Rösler et al. 1999). 
Acknowledgment This study was supported by a research grant of the Swiss National Science foundation to KMR and MRM (Grant 3200B0-100701).

\section{References}

Arányi Z, Mathis J, Hess CW, Rösler KM (1998) Task-dependent facilitation of motor evoked potentials during dynamic and steady muscle contractions. Muscle Nerve 21:1309-1316

Berardelli A, Inghilleri M, Rothwell JC, Cruccu G, Manfredi M (1991) Multiple firing of motoneurones is produced by cortical stimulation but not by direct activation of descending motor tracts. Electroencephalogr Clin Neurophysiol 81:240-242

Bühler R, Magistris MR, Truffert A, Hess CW, Rösler KM (2001) The triple stimulation technique to study central motor conduction to the lower limbs. Clin Neurophysiol 112:938-949

Burke D, Hicks R, Stephen J, Woodforth I, Crawford M (1995) Trialto-trial variability of corticospinal volleys in human subjects. Electroencephalogr Clin Neurophysiol 97:231-237

Day BL, Dressler D, Maertens de Noordhout A, Marsden CD, Nakashima K, Rothwell JC, Thompson PD (1989) Electric and magnetic stimulation of human motor cortex: surface EMG and single motor unit responses. J Physiol (Lond) 412:449-473

Day BL, Rothwell JC, Thompson PD, Dick JPR, Cowan JMA, Berardelli A, Marsden CD (1987) Motor cortex stimulation in intact man. (2) Multiple descending volleys. Brain 110:1191-1209

Ellaway PH, Davey NJ, Maskill DW, Rawlinson SR, Lewis HS, Anissimova NP (1998) Variability in the amplitude of skeletal muscle responses to magnetic stimulation of the motor cortex in man. Electroencephalogr Clin Neurophysiol 109:104-113

Gugino LD, Romero JR, Aglio L, Titone D, Ramirez M, Pascual-Leone A, Grimson E, Weisenfeld N, Kikinis R, Shenton ME (2001) Transcranial magnetic stimulation coregistered with MRI: a comparison of a guided versus blind stimulation technique and its effect on evoked compound muscle action potentials. Clin Neurophysiol 112:1781-1792

Hess CW, Mills KR, Murray NMF (1986) Magnetic stimulation of the human brain: facilitation of motor responses by voluntary contraction of ipsilateral and contralateral muscles with additional observations on an amputee. Neurosci Lett 71:235-240

Humm AM, Z'Graggen WJ, von Hornstein NE, Magistris MR, Rösler KM (2004) Assessment of central motor conduction to intrinsic hand muscles using the triple stimulation technique: normal values and repeatability. Clin Neurophysiol 115:2558-2566

Jankus WR, Robinson LR, Little JW (1994) Normal limits of side-toside H-reflex amplitude variability. Arch Phys Med Rehabil $75: 3-7$
Kiers L, Cros D, Chiappa KH, Fang J (1993) Variability of motor potentials evoked by transcranial magnetic stimulation. Electroencephalogr Clin Neurophysiol 89:415-423

Kischka U, Fajfr R, Fellenberg T, Hess CW (1993) Facilitation of motor evoked potentials from magnetic brain stimulation in man: a comparative study of different target muscles. J Clin Neurophysiol 10:505-512

Magistris MR, Rösler KM, Truffert A, Landis T, Hess CW (1999) A clinical study of motor evoked potentials using a triple stimulation technique. Brain 122:265-279

Magistris MR, Rösler KM, Truffert A, Myers JP (1998) Transcranial stimulation excites virtually all motor neurones supplying the target muscle. A demonstration and a method improving the study of motor evoked potentials. Brain 121:437-450

Naka D, Mills KR (2000) Further evidence for corticomotor hyperexcitability in amyotrophic lateral sclerosis. Muscle Nerve 23:1044-1150

Patton HD, Amassian VE (1954) Single- and multiple-unit analysis of cortical stage of pyramidal tract activation. J Neurophysiol 17:345-363

Rösler KM, Etter C, Truffert A, Hess CW, Magistris MR (1999) Rapid cortical motor output map changes assessed by the triple stimulation technique. NeuroReport 10:579-583

Rösler KM, Truffert A, Hess CW, Magistris MR (2000) Quantification of upper motor neuron loss in amyotrophic lateral sclerosis. Clin Neurophysiol 111:2208-2218

Rossini PM, Desiato MT, Lavaroni F, Caramia MD (1991) Brain excitability and electroencephalographic activation: non-invasive evaluation in healthy humans via transcranial magnetic stimulation. Brain Res 567:111-119

Rothwell JC, Hallett M, Berardelli A, Eisen A, Rossini P, Paulus W (1999) Magnetic stimulation: motor evoked potentials. Electroencephalogr Clin Neurophysiol (Suppl) 52:97-103

Schieppati M, Romano C, Gritti I (1990) Convergence of Ia fibres from synergistic and antagonistic muscles onto interneurones inhibitory to soleus in humans. J Physiol 431:365-377

Ugawa Y, Uesaka Y, Terao Y, Hanajima R, Kanazawa I (1994) Magnetic stimulation of corticospinal pathways at the foramen magnum level in humans. Ann Neurol 36:618-624

Woodforth IJ, Hicks RG, Crawford MR, Stephen JP, Burke DJ (1996) Variability of motor-evoked potentials recorded during nitrous oxide anesthesia from the tibialis anterior muscle after transcranial electrical stimulation. Anesth Analg 82:744-749

Yap CB, Hirota T (1967) Sciatic nerve motor conduction velocity study. J Neurol Neurosurg Psychiatry 30:233-239

Z'Graggen WJ, Humm AM, Durisch N, Magistris MR, Rösler KM (2005) Repetitive spinal motor neuron discharges following single transcranial magnetic stimuli: a quantitative study. Clin Neurophysiol 116:1628-1637 